\title{
Synthesis of Highly Functionalized Piperidines by One-Pot Multicomponent Reaction Using Boron Trifluoride Etherate
}

\author{
A. APPALA NAIDU and G. VEERA RAGHAVA SHARMA* \\ Department of Chemistry, GITAM Institute of Technology, GITAM University \\ Rushikonda, Visakhapatnam-530045, AP, India \\ sharmagvr@yahoo.co.uk
}

Received 21 October 2017 / Accepted 11 November 2017

\begin{abstract}
One-pot, atom and step economic synthesis of highly functionalized piperidines has been developed based on a low-cost and environmentally benign boron trifluoride etherate catalyst. The procedure simply involves the condensation of aromatic aldehydes, $\beta$-ketoesters and aromatic amines in ethanol at room temperature.
\end{abstract}

Keywords: $\mathrm{BF}_{3}$ Etherate, Functionalized piperidines, Aryl aldehyde, One-pot synthesis and characterization

\section{Introduction}

It is known that synthesis of heterocyclic compounds with required biological activity is the major focal challenge in medicinal chemistry and chemical biology. The piperidine and its derivatives are broadly present in many of the natural products, bioactive molecules and organic intermediates ${ }^{1}$. Most of the compounds with piperidine structural moiety exhibit anti-malarial $^{2}$, anti-bacterial ${ }^{3}$, anti-hypertensive ${ }^{4}$, antitumor ${ }^{5}$ and anti-inflammatory ${ }^{6}$ activities. $^{-}$ The piperidine, sub structure is present in some established drugs such as donepezil (1) which is used for treatment of alzheimer's disease, risperidone (2), cyprofloxacine (3) and sertindole (4) used for treatment of schizophrenia (Figure 1).

Nowadays, multicomponent reactions (MCRs) have received huge attention because of the complexity of the molecules that can be easily achieved in one reaction sequence with readily available staring materials. MCRs generally occur in simple, one pot and show high atom economy and product selectivity. In the recent years piperidines are prepared by using catalysts like $\mathrm{InCl}_{3}{ }^{7}$, TFA-proline ${ }^{2}, \mathrm{VCl}_{3}{ }^{8}, \mathrm{ZrOCl}_{2} 8 \mathrm{H}_{2} \mathrm{O}^{9}$, PEG-embedded $\mathrm{KBr}_{3}{ }^{10}$, $\mathrm{Ce}(\mathrm{OTf})_{4}{ }^{11}$, tetrabutylammonium tribromide (TBATB) ${ }^{12}$, bromodimethylsulfonium bromide(BDMS) ${ }^{13}$, phenylboronic acid ${ }^{14}, \mathrm{Ni}\left(\mathrm{ClO}_{4}\right)_{2} \cdot 6 \mathrm{H}_{2} \mathrm{O}^{15}$, Acidic ionic liquids ${ }^{16}$, bismuth nitrate ${ }^{17}$ etc. In the present communication, several functionalized piperidines were synthesized by using Boron trifluoride etherate (Boron trifluoride etherate is used due to its low toxicity and commercial availability). All the synthesized molecules are of potential medicinal/ biological interest and obtained them in excellent yields. 


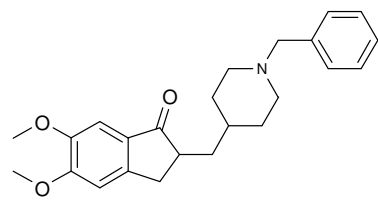

1<smiles>O=C(O)C1CN(C2CC2)c2cc(N3CCNCC3)c(F)cc2C1=O</smiles>

3

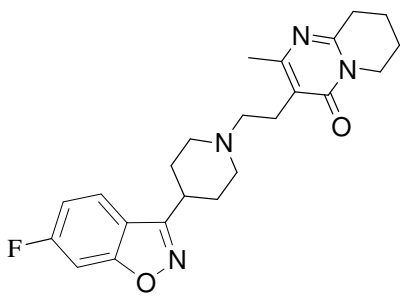

2<smiles>O=C1NCCN1CCN1CCC(C2CN(c3ccc(F)cc3)c3ccc(Cl)cc32)CC1</smiles>

4

Figure 1. Structures of some established drugs with piperidine motif; (1) Donepezil (2) Risperidone (3) Cyprofloxacine and (4) Sertindole

\section{Experimental}

All chemical reagents and solvents were procured from Acros, Fisher scientific and Sigmaaldrich etc., which were directly used as such without any purification. All the reactions were carriedout under inert conditions. The products were isolated through crystalization and precipitation techniques. Percentage of yields were calculated based on pure products. Precoated silica aluminium sheets were used for TLC. The product identified on TLC plate by using Iodine or UV cabinet. NMR spectrum was recorded on Bruker $400 \mathrm{MHz}$ by using solvents $\mathrm{CDCl}_{3}$ or DMSO- $\mathrm{d}_{6}$, mass spectrum on Apex, IR ( $\mathrm{KBr}$ disc) spectrum on Bruker.

In a typical experimental procedure $10 \mathrm{~mol}$ of each aldehyde, amine and $\beta$-keto ester were dissolved in ethanol $(5 \mathrm{~mL}) .10 \mathrm{~mol} \%$ of $\mathrm{BF}_{3}$ etherate was slowly added into reaction mass at $25-35{ }^{\circ} \mathrm{C}$ and maintained for $12-16$ hours at $25-35{ }^{\circ} \mathrm{C}$. Absence of starting materials was monitored by TLC with hexanes and EtOAc mobile phase $(1: 1)$. During maintenance material formation observed, product was collected by simple filtration and wash with ethanol $(1 \mathrm{~mL})$. The material was dried at $50-55{ }^{\circ} \mathrm{C}$. Pale yellow to yellow coloured solid was obtained. The yields are excellent and the obtained product is pure enough without any column purification. The results are briefly mentioned in Table 1. The products were characterized by ${ }^{1} \mathrm{H}$ NMR, ${ }^{13} \mathrm{C}$ NMR, IR and mass etc.

Table 1. Synthesis of functionalized piperidines $(\mathbf{8 a}-\mathbf{g})$ catalyzed by $\mathrm{BF}_{3}$ etherate $(10.0 \mathrm{~mol} \%)$

\begin{tabular}{ccccc}
\hline Entry & R-Arylaldehyde & $\mathrm{R}_{1} \beta$-ketoester & Product & Yield, \% \\
\hline 1 & $\mathrm{H}$ & $\mathrm{CH}_{3}$ & $\mathbf{8 a}$ & 89 \\
2 & $3-\mathrm{F}$ & $\mathrm{CH}_{3}$ & $\mathbf{8 b}$ & 82 \\
3 & $2-\mathrm{F}$ & $\mathrm{CH}_{3}$ & $\mathbf{8 c}$ & 83 \\
4 & $4-\mathrm{F}$ & $\mathrm{CH}_{3}$ & $\mathbf{8 d}$ & 81 \\
5 & $3-\mathrm{OCH}_{3} \& 4-\mathrm{OH}$ & $\mathrm{CH}_{3}$ & $\mathbf{8 e}$ & 87 \\
6 & $4-\mathrm{F}$ & $\mathrm{CH}_{2} \mathrm{CH}_{3}$ & $\mathbf{8 f}$ & 82 \\
7 & $3,4-\mathrm{OCH}_{3}$ & $\mathrm{CH}_{2} \mathrm{CH}_{3}$ & $\mathbf{8 g}$ & 78 \\
\hline
\end{tabular}


Synthesis of methyl 1, 2, 6-triphenyl-4-(phenyl amino)-1, 2, 5, 6-tetrahydropyridine3-carboxylate $(8 a)$

Yield: 89\%. m.p. $191-192{ }^{\circ} \mathrm{C} .{ }^{1} \mathrm{H}$ NMR $\left(400 \mathrm{MHz}, \mathrm{CDCl}_{3}\right): \delta 10.26$ (s, 1H), 7.33-6.45 $(\mathrm{m}, 12 \mathrm{H}), 6.29(\mathrm{~s}, 1 \mathrm{H}), 5.16(\mathrm{t}, \mathrm{J}=7 \mathrm{~Hz}, 1 \mathrm{H}), 3.94(\mathrm{~s}, 3 \mathrm{H}), 2.90(\mathrm{~d}, \mathrm{~J}=7 \mathrm{~Hz}, 2 \mathrm{H}) .{ }^{13} \mathrm{C} \mathrm{NMR}$ $\left(400 \mathrm{MHz}, \mathrm{CDCl}_{3}\right): \delta 168.58,156.26,147.02,144.00,142.79,137.91,128.89-125.77$, 116.22, 113.00, 98.07, 58.23, 55.22, 50.99, 33.65. IR (KBr, cm $\left.{ }^{-1}\right): 3447,3025,2953,1658$, 1253. MS (EI): $\mathrm{m} / \mathrm{z}: 461.2$ and mass fragments are 182.1, 280.1. Anal Calcd. for $\mathrm{C}_{31} \mathrm{H}_{28} \mathrm{~N}_{2} \mathrm{O}_{2}$ : C, 80.84; H, 6.13; N, 6.08; Found: C, 80.65; H, 6.09; N, 6.10.

Synthesis of methyl 2, 6-bis (3-fluorophenyl)-1-phenyl-4-(phenyl amino)-1, 2, 5, 6 tetrahydro pyridine-3-carboxylate $(8 \boldsymbol{b})$

Yield: $82 \%$. m.p. $178-179{ }^{\circ} \mathrm{C} .{ }^{1} \mathrm{H}$ NMR $\left(400 \mathrm{MHz}, \mathrm{CDCl}_{3}\right): \delta 10.64$ (s, 1H), 7.45-6.34 $(\mathrm{m}, 18 \mathrm{H}), 6.12(\mathrm{~s}, 1 \mathrm{H}), 4.57(\mathrm{t}, \mathrm{J}=7 \mathrm{~Hz}, 1 \mathrm{H}), 3.75(\mathrm{~s}, 3 \mathrm{H}), 2.91(2 \mathrm{H}, \mathrm{d}, \mathrm{J}=7 \mathrm{~Hz}),{ }^{13} \mathrm{C}$ NMR $\left(400 \mathrm{MHz}, \mathrm{CDCl}_{3}\right): \delta 168.58,164.54,161.98,157.34,150.18,146.12,146.05,138.42$, 130.33-122.32, 116.87, 115.91, 114.31, 114.09, 113.90, 113.69, 97.03, 61.14, 55.04, 51.1, 36.07. IR $\left(\mathrm{KBr}, \mathrm{cm}^{-1}\right): 3446,3063,2952,1655,1261$. MS (EI): $\mathrm{m} / z: 497.2$ and mass fragments are 200.1, 298.1. Anal Calcd. for $\mathrm{C}_{31} \mathrm{H}_{26} \mathrm{~F}_{2} \mathrm{~N}_{2} \mathrm{O}_{2}$ : C, 74.98; H, 5.28; N, 5.64; Found: C, 74.65; H, 5.25; N, 5.60.

Synthesis of methyl 2, 6-bis (2-fluorophenyl)-1-phenyl-4-(phenyl amino)-1, 2, 5, 6tetrahydro pyridine-3-carboxylate $(8 \mathrm{c})$

Yield: 83\%. m.p. $171-172{ }^{\circ} \mathrm{C} .{ }^{1} \mathrm{H}-\mathrm{NMR}\left(400 \mathrm{MHz}, \mathrm{CDCl}_{3}\right): \delta 10.15$ (s, 1H), 7.23-6.39 $(\mathrm{m}, 18 \mathrm{H}), 6.37(\mathrm{~s}, 1 \mathrm{H}), 5.46(\mathrm{t}, \mathrm{J}=7 \mathrm{~Hz}, 1 \mathrm{H}), 3.89$ (s, 3H), $2.92(\mathrm{~d}, \mathrm{~J}=7 \mathrm{~Hz}, 2 \mathrm{H}) .{ }^{13} \mathrm{C}-\mathrm{NMR}(400$ $\left.\mathrm{MHz}, \mathrm{CDCl}_{3}\right): \delta 168.48,161.93,159.48,155.53,146.39,137.99,129.92-122.38,116.53$, 116.30, 115.27, 115.05, 113.09, 96.84, 52.36, 52.11, 51.02, 31.11. IR (KBr, $\left.\mathrm{cm}^{-1}\right): 3423.4$, 3024.5, 2951.0, 1655.2, 1261.3. MS (EI): $m / z: 497.1$ and mass fragments are 200, 298.1. Anal Calcd. for $\mathrm{C}_{31} \mathrm{H}_{26} \mathrm{~F}_{2} \mathrm{~N}_{2} \mathrm{O}_{2}$ : C, 74.98; H, 5.28; N, 5.64; Found: C, 74.77; H, 5.35; N, 5.68.

Synthesis of methyl 2, 6-bis (4-fluorophenyl)-1-phenyl-4-(phenyl amino)-1, 2, 5, 6tetrahydro pyridine-3-carboxylate $(8 \mathrm{~d})$

Yield: $81 \%$. m.p. $156-157{ }^{\circ} \mathrm{C} .{ }^{1} \mathrm{H}$ NMR $\left(400 \mathrm{MHz}, \mathrm{CDCl}_{3}\right): \delta 10.25$ (s, 1H), 7.30-6.38 $(\mathrm{m}, 18 \mathrm{H}), 6.37(\mathrm{~s}, 1 \mathrm{H}), 5.30(\mathrm{t}, \mathrm{J}=7 \mathrm{~Hz}, 1 \mathrm{H}), 3.93$ (s, 3H), $2.92(\mathrm{~d}, \mathrm{~J}=7 \mathrm{~Hz}, 2 \mathrm{H}) .{ }^{13} \mathrm{C}$ NMR $\left(400 \mathrm{MHz}, \mathrm{CDCl}_{3}\right): \delta 168.36,162.78,160.79,156.05,146.63,139.41,138.12,137.75$, 129.36-124.00, 116.64, 115.66, 115.44, 115.10, 114.89, 113.05, 97.87, 57.32, 54.71, 51.06, 33.79. IR ( $\left.\mathrm{KBr}, \mathrm{cm}^{-1}\right): 3421.3,3046.2,2949.4,1657.7,1267.5$. MS (EI): $\mathrm{m} / z: 497.1$ and mass fragments are 200, 298.1. Anal Calcd. for $\mathrm{C}_{31} \mathrm{H}_{26} \mathrm{~F}_{2} \mathrm{~N}_{2} \mathrm{O}_{2}$ : C, 74.98; H, 5.28; N, 5.64; Found: C, 74.91; H, 5.21; N, 5.63.

Synthesis of methyl 2, 6-bis (4-hydroxy-3-methoxyphenyl)-1-phenyl-4- (phenyl amino)-1, 2, 5, 6-tetrahydro pyridine-3-carboxylate $(8 \boldsymbol{e})$

Yield: 87\%. m.p. $204-205^{\circ} \mathrm{C} .{ }^{1} \mathrm{H}$ NMR (400 MHz, $\left.\mathrm{CDCl}_{3}\right): \delta 10.17$ (s, $\left.1 \mathrm{H}\right), 8.85$ (s, 2H), 7.18$6.42(\mathrm{~m}, 16 \mathrm{H}), 6.14(\mathrm{~s}, 1 \mathrm{H}), 5.20(\mathrm{t}, \mathrm{J}=7 \mathrm{~Hz}, 1 \mathrm{H}), 3.84(\mathrm{~s}, 3 \mathrm{H}), 3.66(\mathrm{~s}, 3 \mathrm{H}), 3.58(\mathrm{~s}, 3 \mathrm{H}), 2.95$ $(\mathrm{d}, \mathrm{J}=7 \mathrm{~Hz}, 2 \mathrm{H}) .{ }^{13} \mathrm{C} \mathrm{NMR}\left(400 \mathrm{MHz}, \mathrm{CDCl}_{3}\right): \delta 167.65,155.07,147.60,147.42,146.58$, $145.40,145.05,137.81,134.95,133.55,128.87-124.86,118.53,118.30,115.50-115.24$, $112.48,110.55,97.92,56.24,55.50,55.45,54.77,50.91,33.53$. IR $\left(\mathrm{KBr}, \mathrm{cm}^{-1}\right): 3398.0$, 3020.7, 2958.8, 1641.5, 1267.8. MS (EI): $\mathrm{m} / \mathrm{z}: 553.2$ and mass fragments are 228.1, 326.1. Anal Calcd. for $\mathrm{C}_{33} \mathrm{H}_{32} \mathrm{~N}_{2} \mathrm{O}_{6}$ : C, 71.72; H, 5.84; N, 5.07; Found: C, 71.69; H, 5.89; N, 5.10. 
Synthesis of ethyl 2, 6-bis (4-fluorophenyl)-1-phenyl-4-(phenyl amino)-1, 2, 5, 6tetrahydropyridine-3-carboxylate $(8 \mathrm{f})$

Yield: 82\%. m.p. 202-204 ${ }^{\circ} \mathrm{C} .{ }^{1} \mathrm{H}$ NMR (400 MHz, $\mathrm{CDCl}_{3}$ ): $\delta 10.32$ (s, 1H), 7.30-6.41 (m, 18H), 6.39 (s, 1H), $5.12(\mathrm{t}, \mathrm{J}=7 \mathrm{~Hz}, 1 \mathrm{H}), 4.48-4.26(\mathrm{~m}, 2 \mathrm{H}), 2.86$ (d, J=7 Hz, 2H), 1.48 $(\mathrm{t}, \mathrm{J}=8 \mathrm{~Hz}, 3 \mathrm{H}) .{ }^{13} \mathrm{C} \mathrm{NMR}\left(400 \mathrm{MHz}, \mathrm{CDCl}_{3}\right): \delta 168.07,163.25,160.82,155.87,146.93$, $139.59,138.19,137.94,129.02-125.67,116.66,115.57,115.36,115.12,114.89,113.13$, 98.19, 60.90, 58.04, 55.85, 36.69, 14.80. IR $\left(\mathrm{KBr}, \mathrm{cm}^{-1}\right): 3415.8,3059.5,2978.8,1651.2$, 1257.1. MS (EI): $\mathrm{m} / \mathrm{z}: 511.1$ and mass fragments are 200.1, 312.1. Anal Calcd. for $\mathrm{C}_{32} \mathrm{H}_{28} \mathrm{~F}_{2} \mathrm{~N}_{2} \mathrm{O}_{2}$ : C, 75.28; H, 5.53; N, 5.49; Found: C, 75.15; H, 5.49; N, 5.39.

Synthesis of ethyl 2, 6-bis (3, 4-dimethoxyphenyl)-1-phenyl-4-(phenyl amino)-1, 2, 5, 6-tetrahydropyridine-3-carboxylate $(8 \mathrm{~g})$

Yield: 78\%. m.p. $162-164{ }^{\circ} \mathrm{C} .{ }^{1} \mathrm{H}$ NMR $\left(400 \mathrm{MHz}, \mathrm{CDCl}_{3}\right): \delta 10.32(\mathrm{~s}, 1 \mathrm{H}), 7.26-6.38(\mathrm{~m}$, $16 \mathrm{H}), 6.35$ (s, 1H), $5.06(\mathrm{t}, \mathrm{J}=7 \mathrm{~Hz}, 1 \mathrm{H}), 4.48-4.25(\mathrm{~m}, 2 \mathrm{H}), 3.90-3.70$ (s, 12H), 2.96 (d, J=7 $\mathrm{Hz}, 2 \mathrm{H}), 1.46(\mathrm{t}, \mathrm{J}=8 \mathrm{~Hz}, 3 \mathrm{H}) .{ }^{13} \mathrm{C}-\mathrm{NMR}\left(400 \mathrm{MHz}, \mathrm{CDCl}_{3}\right): \delta 167.23,155.16,148.81-147.40$, 146.47, 137.73, 136.55, 135.11, 129.72-124.80, 118.27, 117.95, 115.62-110.10, 97.84, 59.33, 56.19, 55.89, 55.61, 55.56, 55.35, 54.69, 33.43, 14.68. IR $\left(\mathrm{KBr}, \mathrm{cm}^{-1}\right): \mathrm{cm}^{-1} 3415.9,3190$, 2933.5, 1652.9, 1255.3. MS (EI): $\mathrm{m} / z: 595.1$ and mass fragments are 242.1, 354.1. Anal Calcd. for $\mathrm{C}_{36} \mathrm{H}_{38} \mathrm{~N}_{2} \mathrm{O}_{6}: \mathrm{C}, 72.71 ; \mathrm{H}, 6.44 ; \mathrm{N}, 4.71$; Found: C, 72.69; H, 6.39; N, 4.59.

Synthesis of methyl 5-(2'-butyl-4'-chloro-1'H-imidazol-5'-yl)-2, 7-dimethyl-5Hthiazolo [3, 2-a] pyrimidine-6-carboxylate (17)

It is the reaction of 2-butyl-4-chloro- $1 \mathrm{H}$-imidazole-5-carbaldehyde with 2-amino-5-methyl thiazole and methylacetoacetate, follows above experimental procedure but after 12 hours no material formation observed, material formation observed after 24 hours. The product obtained as solid. Yield is about $74 \%$. m.p. $216-218^{\circ} \mathrm{C}$. The spectral data is obtained as shown below. ${ }^{1} \mathrm{H}$ NMR (400 MHz, $\left.\mathrm{CDCl}_{3}\right): \delta 13.56(\mathrm{~s}, 1 \mathrm{H}), 6.37(\mathrm{~s}, 2 \mathrm{H}), 3.65(\mathrm{~s}, 3 \mathrm{H}), 2.63(\mathrm{t}, \mathrm{J}=7 \mathrm{~Hz}, 2 \mathrm{H})$, $2.24(\mathrm{~s}, 3 \mathrm{H}), 2.11(\mathrm{~s}, 3 \mathrm{H}), 1.71-1.65(\mathrm{~m}, 2 \mathrm{H}), 1.35-1.30(\mathrm{~m}, 2 \mathrm{H}), 0.89(\mathrm{t}, \mathrm{J}=8 \mathrm{~Hz}, 3 \mathrm{H})$. ${ }^{13} \mathrm{C}$ NMR $\left(400 \mathrm{MHz}, \mathrm{CDCl}_{3}\right): \delta 166.36,164.22,155.09,149.59,126.42,124.64,122.78$, 119.63, 97.32, 51.24, 50.94, 30.36, 28.58, 22.51, 22.44, 13.73, 12.78. IR (KBr, $\left.\mathrm{cm}^{-1}\right): 3421.4$, 3037.2, 2926.1, 1698.3, 1614.8, 1578, 1427, 1301.2, 1092.2. MS (EI): $\mathrm{m} / z:$ 381.1. Anal Calcd. for $\mathrm{C}_{17} \mathrm{H}_{21} \mathrm{ClN}_{4} \mathrm{O}_{2} \mathrm{~S}$ : C, 53.61; H, 5.56; N, 14.71; Found: C, 53.65; H, 5.55; N, 14.61 .

\section{Results and Discussion}

We have synthesized novel piperidine derivatives by the reaction of aromatic aldehyde, amine and $\beta$-ketoester using readily available Lewis acid catalyst i.e., boron trifluoride etherate represented in the Scheme 1.

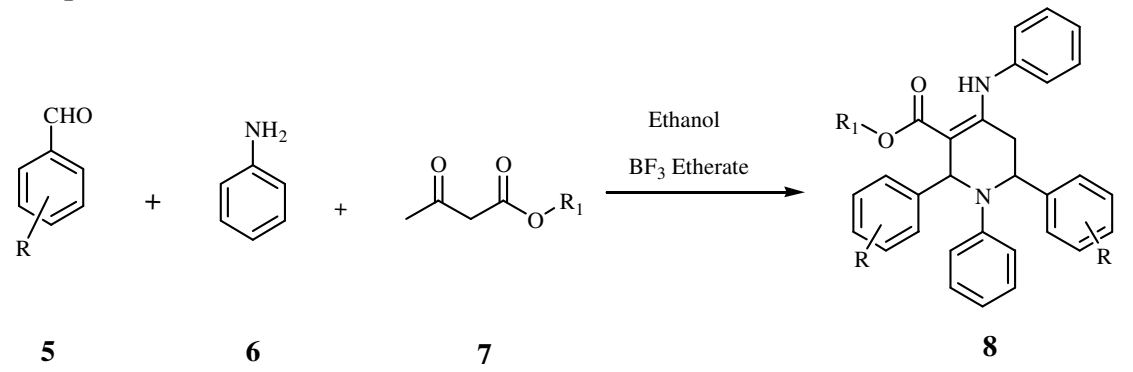

Scheme 1. Synthesis of functionalized piperidines 
Initially we have carried out the reaction with $10 \mathrm{~mol}$ of each benzaldehyde, aniline and methyl acetoacetate to optimize the reaction conditions. In many publications reaction not occurs in neat conditions, hence we optimize the reaction conditions by using boron trifluoride etherate as catalyst in different solvents such as methanol, ethanol, acetonitrile and dichloromethane. Among these ethanol is the best solvent and gives the good yield at ambient temperature. We tried the reaction with $p$-nitro aniline but reaction not occurs due to the reduced nucleophilicity.

We observe that in all mass spectrums of compounds $\mathbf{9}$ and $\mathbf{1 0}$ the mass fragments are shown in Figure 2. Based on the those mass fragments finally we proposed a plausible mechanism in Scheme 2, in which aryl aldehyde reacts with enamine, which was formed initially from the condensation of aniline with $\beta$-ketoester and the resulting probable Knovenagelproduct $\mathbf{1 2}$ or $\mathbf{1 3}$ was then reacts with pure imine $\mathbf{9}$ (obtained from the reaction of aniline and aldehyde ), and eventually generating $\mathbf{8}$.<smiles>C(=N/c1ccccc1)\c1ccccc1</smiles><smiles>[R]OC(=O)/C(=C\c1ccccc1)C(=C)Nc1ccccc1</smiles>

10

Figure 2. Mass fragments of compound 8

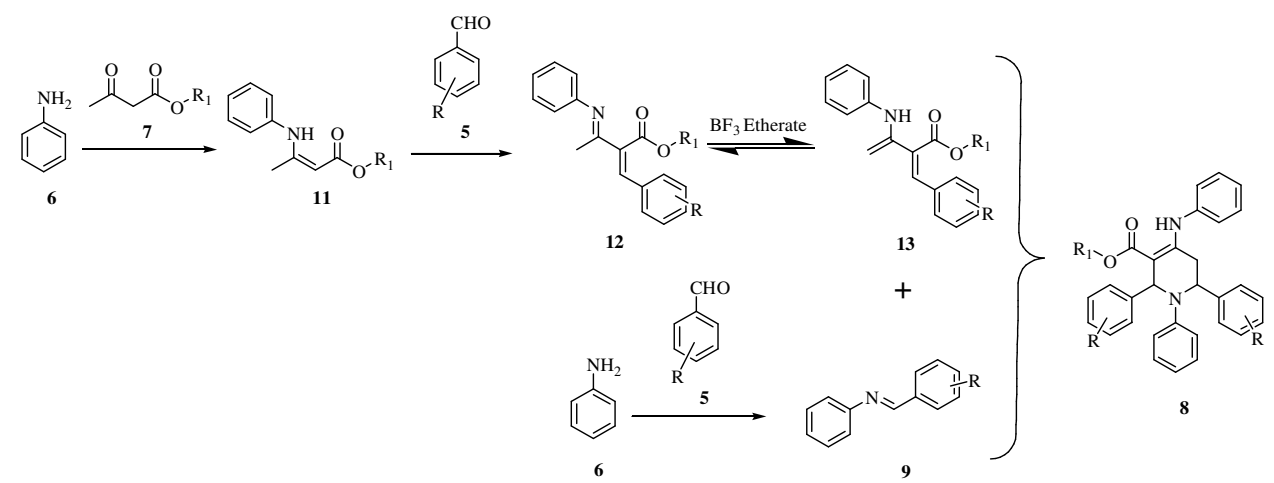

Scheme 2. Plausible mechanism for the synthesis of piperidines

In the similar fashion, reaction of immidazole aldehyde i.e., 2-butyl-4-chloro- $1 \mathrm{H}$ imidazole-5-carbaldehyde (14) with 2-amino-5-methyl thiazole (15) and methyl acetoacetate (16) in presence of $\mathrm{BF}_{3}$ etherate in ethanol solvent yields novel thiazolopyrimidine derivative 17 instead of expected piperidine derivative, reaction Scheme 3 is mentioned below.<smiles>Cc1nc(Cl)c(C=[OH+])[nH]1</smiles><smiles>COC(=O)C1=C(C)N=C2SC(C)=CN2C1c1[nH]c(SC)nc1Cl</smiles>

14 15 16 17

Scheme 3. Synthesis of novel thiazolopyrimidine derivative instead of expected piperidine 


\section{Conclusion}

We have developed a very simple, practical and easily scalable, multicomponent method for the synthesis of piperidine derivatives with commercially available inexpensive lewis acid catalyst i.e. boron trifluoride etherate with high atom economy. All the piperidine derivatives are obtained in good yield by simple filtration, without any further purification. Due to easy availabilty of the starting materials, the reaction might prove to be very useful for building up piperdine motifs. All the obtained products were well characterized by the spectral techniques.

\section{Acknowledgement}

We thank Laila-Impex, Vijayawada and Institute of Life Sciences, University of Hyderabad, Hyderabad for the spectral data. This work is part of Ph.D. thesis of A. Appala Naidu. A. Appala Naidu is grateful to Vijayasree Organics for the encouragement. We acknowledge the financial support of DST-FIST to Department of Chemistry, GITAM University and TEQIP to GITAM Institute of Technology.

\section{References}

1. Watson P S, Jiang B and Scott B, Org Lett., 2000, 2(23), 3679-3682; DOI:10.1021/ol006589o

2. Misra M, Pandey S K, Pandey V P, Pandey J, Tripathi R and Tripathi R P, Bioorg Med Chem., 2009, 17(2), 625-633; DOI:10.1016/j.bmc.2008.11.062

3. Zhou Y, Gregor V E, Ayida B K, Winters G C, Sun Z, Murphy D, Haley G, Bailey D, Froelich J M, Fish S, Webber S E, Hermann T and Wall D, Bioorg Med Chem Lett., 2007, 17(5), 1206-1210; DOI:10.1016/j.bmcl.2006.12.024

4. Petit S, Nallet J P, Guillard M, Dreux J, Chermat R, Poncelet M, Bulach C, Simon P, Fontaine C, Barthelmebs M and Imbs J L, Eur J Med Chem., 1991, 26(1), 19-32; DOI:10.1016/0223-5234(91)90209-6

5. Liu X H, Li J, Shi J B, Song B A and Qi X B, Eur J Med Chem., 2012, 51, 294-299; DOI:10.1016/j.ejmech.2012.02.040

6. Bin H, Crider A M and Stables J P, Eur J Med Chem., 2001, 36(3), 265-286; DOI:10.1016/S0223-5234(00)01206-X

7. Clarke P A, Zaytzev A V and Whitwood A C, Tetrahedron Lett., 2007, 48(30), 52095212; DOI:10.1016/j.tetlet.2007.05.141

8. Pal S, Choudhury L H and Parvin T, Mol Divers, 2012, 16(1), 129-143; DOI:10.1007/s11030-011-9339-9

9. Mishra S and Ghosh R, Tetrahedron Lett., 2011, 52(22), 2857-2861; DOI:10.1016/j.tetlet.2011.03.116

10. Sanny V, Jai S L and Sain B, Beilstein J Org Chem., 2011, 7, 1334-1341; DOI:10.3762/bjoc.7.157

11. Samad K, Seyed M V and Mandana A, Comptes Rendus Chimie, 2013, 16(11), 10241028; DOI:10.1016/j.crci.2013.03.011

12. Khan A T, Lal M and Khan M M, Tetrahedron Lett., 2010, 51(33), 4419-4424; DOI:10.1016/j.tetlet.2010.06.069

13. Khan A T, Parvin T and Choudhury L H, J Org Chem., 2008, 73(21), 8398-8402; DOI:10.1021/jo8014962

14. Goswami S V, Thorat P B and Bhusare S R, Heterocyclic Commun., 2012, 18(5-6), 248-254; DOI:10.1515/hc-2012-0105 
15. Brahmachari G and Das S, J Org Biomol Chem., 2013, 1, 33-46.

16. Hamid R S and Kobra A, J Molecular Liquids, 2013, 180, 187-191; DOI:10.1016/j.molliq.2013.01.020

17. Brahmachari $G$ and Das S, Tetrahedron Lett., 2012, 53(12), 1479-1484; DOI:10.1016/j.tetlet.2012.01.042 